\title{
Performance of Synthetic Double Sampling Chart with Estimated Parameters Based on Expected Average Run Length
}

\author{
Huay Woon You (iD \\ Pusat PERMATApintar Negara, Universiti Kebangsaan Malaysia, 43600 UKM Bangi, Selangor, Malaysia \\ Correspondence should be addressed to Huay Woon You; hwyou@ukm.edu.my
}

Received 10 February 2018; Revised 11 April 2018; Accepted 23 April 2018; Published 31 May 2018

Academic Editor: Dejian Lai

Copyright (C) 2018 Huay Woon You. This is an open access article distributed under the Creative Commons Attribution License, which permits unrestricted use, distribution, and reproduction in any medium, provided the original work is properly cited.

\begin{abstract}
A synthetic double sampling (SDS) chart is commonly evaluated based on the assumption that process parameters (namely, mean and standard deviation) are known. However, the process parameters are usually unknown and must be estimated from an incontrol Phase-I dataset. This will lead to deterioration in the performance of a control chart. The average run length (ARL) has been implemented as the common performance measure in process monitoring of the SDS chart. Computation of ARL requires practitioners to determine shift size in advance. However, this requirement is too restricted as practitioners may not have the experience to specify the shift size in advance. Thus, the expected average run length (EARL) is introduced to assess the performance of the SDS chart when the shift size is random. In this paper, the SDS chart, with known and estimated process parameters, was evaluated based on EARL and compared with the performance measure, ARL.
\end{abstract}

\section{Introduction}

The quality of products and services is an essential factor in the world of business [1]. In order to enhance the quality of products and services, statistical process control (SPC) is used to monitor and attain the process of manufacturing and services. Among the SPC techniques, control charts are one of the most prominent techniques for detecting shifts in a process. The first control chart was proposed by Dr. Walter A. Shewhart, and it was named the Shewhart chart. The Shewhart chart is frequently used to detect large process mean shifts due to its simplicity [2]. However, the main limitation of the Shewhart chart is that it is insensitive in detecting moderate and small process mean shifts.

In recent years, Khoo et al. [3] suggested synthetic double sampling (SDS) chart, which combines double sampling (DS) subchart and conforming run length (CRL) subchart. From the findings, the SDS chart is efficient in detecting moderate and small process mean shifts compared to the synthetic chart and double sampling chart. The implementation of the SDS chart is based on the assumption that the process parameters are known. Nevertheless, the process parameters are generally unknown in practice. This requires for an estimation of the process parameters from the in-control Phase-I samples.

Saleh and Mahmoud [4] claimed that when the estimated process parameters are used in place of the known process parameters, the performance of the control chart is affected due to the existence of variability in the estimation. Woodall and Montgomery [5] recognised the importance of examining the effects of parameter estimation on the performance of a particular control chart. Therefore, the effects of parameter estimation need to be considered when designing a control chart. Among others, [6-8] have examined the impacts of Phase-I parameter estimation on the performance of a control chart.

The performance of a control chart is crucial in determining the appropriate control chart to be implemented in a process. A common performance measure in process monitoring is average run length (ARL). ARL is the number of samples (on average) plotted on a control chart before it signals an out-of-control [9]. By using ARL as a performance measure, the chart's user needs to determine the process shift size.

You et al. [10] investigated the ARL performance of the SDS chart when process parameters were estimated and this 
has motivated the current research work. In most practical situations, it is usual that the next shift size is unknown in advance $[11,12]$. To circumvent this problem, the expected average run length (EARL) is proposed to examine the performance of the SDS chart when the process shift size is unknown and random. Hence, the performance of the SDS chart, with known and estimated process parameters, is investigated using EARL. In addition, the proposed alternative performance measure, i.e., EARL of the SDS chart, will be compared with the corresponding chart using ARL.

The rest of this paper is structured as follows: Section 2 presents the operation and steps to implement the SDS chart. Moreover, the run length properties of the SDS chart with known and estimated process parameters are also given in Section 2. Section 3 illustrates the performance comparison of the SDS chart, based on EARL and ARL, for known process parameters and that of the corresponding chart with estimated process parameters. Finally, concluding remarks are drawn in the last section.

\section{Materials and Methods}

Khoo et al. [3] developed the SDS chart, which comprises the DS subchart and a CRL subchart. The CRL subchart is an attribute chart, with one lower limit, i.e., $L_{3}$. Figure 1 illustrates the operation of the SDS chart with known process parameters.

Step 1. Set the charting parameters $n_{1}, n_{2}, L, L_{1}, L_{2}$, and $L_{3}$.

Step 2. At sampling time $i=1,2, \ldots$, take the first sample of size $n_{1}$ and the sample mean, $\bar{Y}_{1, i}=\sum_{j=1}^{n_{1}} Y_{1 j} / n_{1}$, is calculated.

Step 3. Compute the standardised statistic $Z_{1, i}=\left[\left(\bar{Y}_{1, i}-\right.\right.$ $\left.\left.\mu_{0}\right) \sqrt{n_{1}}\right] / \sigma_{0}$ corresponding to the first sample.

Step 4. If $Z_{1, i}$ is in $I_{1}$, the $i$ th sampling time is conforming and the control flow returns to Step 2.

Step 5. If $Z_{1, i}$ is in $I_{3}$, the $i$ th sampling time is nonconforming and the control flow goes to Step 9 .

Step 6. If $Z_{1, i}$ is in $I_{2}$, take a second sample with $n_{2}$ and compute the sample mean, $\bar{Y}_{2, i}=\sum_{j=1}^{n_{2}} Y_{2 j} / n_{2}$.

Step 7. Calculate the sample mean of the combined samples $\bar{Y}_{i}=\left(n_{1} \bar{Y}_{1, i}+n_{2} \bar{Y}_{2, i}\right) /\left(n_{1}+n_{2}\right)$ and the standardised statistic $Z_{i}=\left[\left(\bar{Y}_{i}-\mu_{0}\right) \sqrt{n_{1}+n_{2}}\right] / \sigma_{0}$ corresponding to the combined samples.

Step 8. If $Z_{i}$ is in $I_{4}$, and the $i$ th sampling time is conforming, then return to Step 2. Otherwise, the sampling time is regarded as nonconforming and the control flow goes to Step 9 .

Step 9. Count the number of inspected sampling times between the present and last nonconforming sampling times inclusive of the present nonconforming sampling time, and denote it as the CRL value.

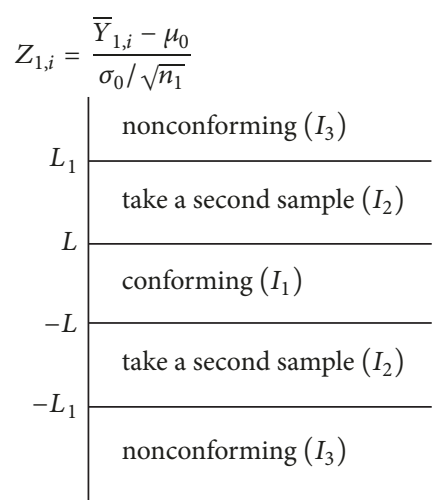

(a) First sample

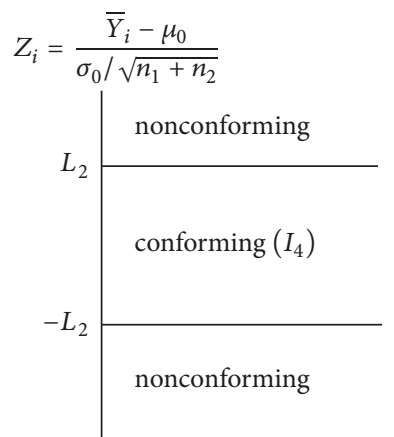

(b) Combined samples
FiguRE 1: DS subchart.

Step 10. If CRL $>L_{3}$, the process is in-control and the control flow returns to Step 2. Otherwise, the process is outof-control and immediate actions are required to eliminate the assignable cause(s). Then, return to Step 2.

Without loss of generality, the in-control mean, $\mu_{0}$, and in-control standard deviation, $\sigma_{0}$, are assumed as known. Let $P=1-P_{a}-P_{b}$ be the probability of deciding that a sampling time is nonconforming in the DS subchart. Note that $P_{a}$ and $P_{b}$ can be expressed as follows [3]:

$$
\begin{aligned}
P_{a} & =\operatorname{Pr}\left(Z_{1, i} \in I_{1}\right)=\Phi\left(L+\delta \sqrt{n_{1}}\right)-\Phi\left(-L+\delta \sqrt{n_{1}}\right), \\
P_{b} & =\operatorname{Pr}\left(Z_{i} \in I_{4}, Z_{1, i} \in I_{2}\right) \\
& =\int_{z \in I_{2}^{*}}\left[\Phi\left(c L_{2}+r c \delta-z \sqrt{\frac{n_{1}}{n_{2}}}\right)\right. \\
& \left.-\Phi\left(-c L_{2}+r c \delta-z \sqrt{\frac{n_{1}}{n_{2}}}\right)\right] \phi(z) d z,
\end{aligned}
$$

where $\Phi(\cdot)$ and $\phi(\cdot)$ are the cumulative distribution function (cdf) and probability density function (pdf) for a standard normal random variable, respectively. Here, $r=\sqrt{n_{1}+n_{2}}$, $c=r / \sqrt{n_{2}}$, and $I_{2}^{*}=\left[-L_{1}+\delta \sqrt{n_{1}},-L+\delta \sqrt{n_{1}}\right) \cup\left(L+\delta \sqrt{n_{1}}, L_{1}+\right.$ $\left.\delta \sqrt{n_{1}}\right]$.

Finally, for the SDS chart when process parameters are known, the ARL is equal to

$$
\mathrm{ARL}=\frac{1}{P} \times \frac{1}{1-(1-P)^{L_{3}}}
$$

Moreover, when the exact shift size is unknown, it is essential to consider the EARL for an overall range of shifts $\left(\delta_{\min }, \delta_{\max }\right)$, where $\delta_{\min }$ and $\delta_{\max }$ indicate the lower and upper bounds of the mean shift, respectively. The EARL of the SDS chart with known process parameters is

$$
\mathrm{EARL}=\int_{\delta_{\min }}^{\delta_{\max }} f_{\delta}(\delta) \mathrm{ARL} \mathrm{d} \delta,
$$

where ARL can be obtained from $(2)$ and $f_{\delta}(\delta)$ is the pdf of the shift size $\delta$. Since the actual shape of $f_{\delta}(\delta)$ is usually 
unknown, it is assumed that the shifts in process mean happen with equal probability; i.e., $\delta$ is uniformly distributed with $U\left(\delta_{\min }, \delta_{\max }\right)$. Therefore, (3) reduces to

$$
\mathrm{EARL}=\frac{1}{\delta_{\max }-\delta_{\min }} \int_{\delta_{\min }}^{\delta_{\max }} \operatorname{ARL} d \delta
$$

In reality, $\mu_{0}$ and $\sigma_{0}$ are unknown and need to be estimated from $m$ Phase-I samples, each of size $n$, i.e., $\left\{X_{i, 1}, X_{i, 2}, \ldots, X_{i, n}\right\}$, for $i=1,2, \ldots, m$. The estimators of $\mu_{0}$ and $\sigma_{0}$ are $[10]$

$$
\begin{aligned}
& \widehat{\mu}_{0}=\frac{1}{m n} \sum_{i=1}^{m} \sum_{j=1}^{n} X_{i, j}, \\
& \widehat{\sigma}_{0}=\sqrt{\frac{1}{m(n-1)} \sum_{i=1}^{m} \sum_{j=1}^{n}\left(X_{i, j}-\bar{X}_{i}\right)^{2}},
\end{aligned}
$$

respectively. As the values of $\mu_{0}$ and $\sigma_{0}$ are both unknown and need to be estimated using $\widehat{\mu}_{0}$ and $\widehat{\sigma}_{0}$, the standardised statistic for the first sample and combined samples at the sampling time $i$ of the DS subchart with estimated process parameters becomes

$$
\begin{aligned}
\widehat{Z}_{1, i} & =\frac{\bar{X}_{1, i}-\widehat{\mu}_{0}}{\widehat{\sigma}_{0} / \sqrt{n_{1}^{\prime}}} \\
\widehat{Z}_{i} & =\frac{\bar{X}_{i}-\widehat{\mu}_{0}}{\widehat{\sigma}_{0} / \sqrt{n_{1}^{\prime}+n_{2}^{\prime}}}
\end{aligned}
$$

respectively.

Here, $n_{1}^{\prime}$ and $n_{2}^{\prime}$ represent the sample sizes of the SDS chart with the estimated process parameters. Similarly, $L^{\prime}, L_{1}^{\prime}, L_{2}^{\prime}$, and $L_{3}^{\prime}$ correspond to the limits of the SDS chart when the process parameters are estimated.

Then, the probability for the DS subchart with the estimated process parameters to identify a nonconforming sampling time is given as $\widehat{P}=1-\widehat{P}_{a}-\widehat{P}_{b}$; i.e., [10],

$$
\begin{aligned}
\widehat{P}= & 1-\left[\Phi\left(W \sqrt{\frac{n_{1}^{\prime}}{m n}}+R L^{\prime}-\delta \sqrt{n_{1}^{\prime}}\right)\right. \\
& \left.-\Phi\left(W \sqrt{\frac{n_{1}^{\prime}}{m n}}-R L^{\prime}-\delta \sqrt{n_{1}^{\prime}}\right)\right] \\
& -\left(\int_{z \in I_{2}} \widehat{P}_{4} \times f_{\widehat{Z}_{1, i}}\left(z \mid \widehat{\mu}_{0}, \widehat{\sigma}_{0}\right) d z\right),
\end{aligned}
$$

with

$$
\begin{aligned}
& \widehat{P}_{4}=\Phi\left[W \sqrt{\frac{n_{2}^{\prime}}{m n}}+R\left(\frac{L_{2}^{\prime} \sqrt{n_{1}^{\prime}+n_{2}^{\prime}}-z \sqrt{n_{1}^{\prime}}}{\sqrt{n_{2}^{\prime}}}\right)\right. \\
& \left.-\delta \sqrt{n_{2}^{\prime}}\right]-\Phi\left[W \sqrt{\frac{n_{2}^{\prime}}{m n}}\right. \\
& \left.-R\left(\frac{L_{2}^{\prime} \sqrt{n_{1}^{\prime}+n_{2}^{\prime}}+z \sqrt{n_{1}^{\prime}}}{\sqrt{n_{2}^{\prime}}}\right)-\delta \sqrt{n_{2}^{\prime}}\right] \text {, } \\
& f_{\widehat{Z}_{1, i}}\left(z \mid \widehat{\mu}_{0}, \widehat{\sigma}_{0}\right)=R \phi\left(W \sqrt{\frac{n_{1}^{\prime}}{m n}}+R z-\delta \sqrt{n_{1}^{\prime}}\right) .
\end{aligned}
$$

Here, $W$ and $R$ are random variables denoted as

$$
\begin{aligned}
& W=\left(\widehat{\mu}_{0}-\mu_{0}\right) \frac{\sqrt{m n}}{\sigma_{0}}, \\
& R=\frac{\widehat{\sigma}_{0}}{\sigma_{0}},
\end{aligned}
$$

respectively. $W$ is

As $\widehat{\mu}_{0} \sim N\left[\mu_{0}, \sigma_{0}^{2} /(m n)\right]$, it can be deduced that the pdf of

$$
f_{W}(w)=\phi(w)
$$

For the random variable $R$, it is known that $\widehat{\sigma}_{0}^{2} / \sigma_{0}^{2} \sim$ $\gamma(m(n-1) / 2,2 /(m(n-1)))$, i.e., the gamma distribution with parameters $[m(n-1)] / 2$ and $2 /[m(n-1)]$. Using this property, the pdf of $R$ is as follows:

$$
f_{R}(r \mid m, n)=2 r f_{\gamma}\left(r^{2} \mid \frac{m(n-1)}{2}, \frac{2}{m(n-1)}\right),
$$

where $f_{\gamma}(\cdot)$ is the pdf of the gamma distribution with parameters $[m(n-1)] / 2$ and $2 /[m(n-1)]$. Note that, for complete and detailed derivation, reader can refer to You et al. [10].

Thus, the ARL of the SDS chart with estimated process parameters is

$$
\begin{aligned}
& \mathrm{ARL}_{m}=\int_{-\infty}^{+\infty} \int_{0}^{+\infty} \frac{1}{\widehat{P}} \times \frac{1}{1-(1-\widehat{P})^{L_{3}^{\prime}}} f_{W}(w) \\
& \cdot f_{R}(r \mid m, n) d r d w .
\end{aligned}
$$

Consequently, when the process parameters are estimated from the in-control Phase-I samples, the computation of the EARL is

$$
\begin{aligned}
& \operatorname{EARL}_{m} \\
& =\int_{-\infty}^{+\infty} \int_{0}^{+\infty} \operatorname{EARL} f_{W}(w) f_{R}(r \mid m, n) d r d w,
\end{aligned}
$$

where EARL can be obtained from (3) by replacing $P$ and $L_{3}$ with $\widehat{P}$ and $L_{3}^{\prime}$, respectively. 
TABLE 1: Optimal charting parameters $\left(n_{1}, n_{2}, L, L_{1}, L_{2}, L_{3}\right)$ and the corresponding EARL $\mathrm{E}_{1}$ for $n=\{3,4,5,6\}$ with different combinations of $\left(m, \delta_{\min }, \delta_{\max }\right)$ when $\mathrm{EARL}_{0}=370.4$.

\begin{tabular}{ccccccccccccccc}
\hline$n$ & $\delta_{\min }$ & $\delta_{\max }$ & $n_{1}$ & $n_{2}$ & $L$ & $L_{1}$ & $L_{2}$ & $L_{3}$ & 30 & 50 & 80 & 200 & 500 & $+\infty$ \\
\hline 3 & 0.2 & 1.0 & 2 & 6 & 1.3830 & 5.2804 & 2.0239 & 9 & 3.56 & 3.36 & 3.26 & 3.18 & 3.15 & 3.13 \\
& 1.0 & 2.0 & 2 & 2 & 0.6745 & 4.8576 & 2.0503 & 2 & 1.02 & 1.02 & 1.02 & 1.01 & 1.01 & 1.01 \\
4 & 0.2 & 1.0 & 3 & 6 & 1.3830 & 5.2804 & 2.0539 & 7 & 2.60 & 2.51 & 2.46 & 2.42 & 2.40 & 2.40 \\
& 1.0 & 2.0 & 3 & 3 & 0.9674 & 4.9920 & 2.0086 & 2 & 1.00 & 1.00 & 1.00 & 1.00 & 1.00 & 1.00 \\
5 & 0.2 & 1.0 & 3 & 8 & 1.1503 & 5.0443 & 2.0618 & 6 & 2.05 & 2.00 & 1.98 & 1.96 & 1.96 & 1.95 \\
& 1.0 & 2.0 & 4 & 3 & 0.9674 & 4.9920 & 2.0363 & 2 & 1.00 & 1.00 & 1.00 & 1.00 & 1.00 & 1.00 \\
6 & 0.2 & 1.0 & 4 & 9 & 1.2206 & 5.1630 & 2.0210 & 5 & 1.73 & 1.71 & 1.69 & 1.68 & 1.68 & 1.67 \\
& 1.0 & 2.0 & 5 & 3 & 0.9674 & 4.9920 & 2.0527 & 2 & 1.00 & 1.00 & 1.00 & 1.00 & 1.00 & 1.00 \\
\hline
\end{tabular}

\section{Results and Discussion}

In practice, the exact shift size of a process is unknown. In this situation, if the corresponding optimal charting parameters are employed based on a particular shift size, the performance of the control chart will be significantly different if different shift occurred in the process. Therefore, it is essential to evaluate the performance of the SDS chart using alternative performance measure, i.e., EARL. In this paper, the optimal charting parameters $\left(n_{1}, n_{2}, L, L_{1}, L_{2}, L_{3}\right)$ of the SDS chart were computed using a nonlinear minimisation problem, i.e., optimal statistical design that minimises the out-of-control EARL $\left(\mathrm{EARL}_{1}\right)$. The programmes are written in the ScicosLab software version 4.4.2 (http://www.scicoslab.org).

The optimal charting parameters and the corresponding EARL $_{1} s$ with different combinations of sample size, $n=\{3,4,5,6\}$, the number of Phase-I samples, $m=$ $\{30,50,80,200,500,+\infty\}$ with $\left(\delta_{\min }, \delta_{\max }\right)=(0.2,1.0)$ and $\left(\delta_{\min }, \delta_{\max }\right)=(1.0,2.0)$ with the in-control EARL, i.e., $\mathrm{EARL}_{0}=370.4$, are presented in Table 1 . Here, $m=+\infty$ denotes the known process parameters case, while $m=$ $\{30,50,80,200,500\}$ denotes the estimated process parameters case. The performance of the SDS chart for both the known and estimated process parameters cases was calculated using the optimal charting parameters in columns 4-9, which were obtained by minimising EARL $\mathrm{E}_{1}$ when the process parameters are known.

From Table 1 , for the same $n, \delta_{\min }$, and $\delta_{\max }$, the value of $\mathrm{EARL}_{1}$ decreases with the increases in $m$. This is due to the fact that as the more Phase-I samples are taken, the performance of the estimated process parameters SDS chart approaches to the corresponding chart with known process parameters; i.e., $\mathrm{EARL}_{1}$ value decreases to indicate better performance. For instance, when $n=3, \delta_{\min }=$ 0.2 , and $\delta_{\max }=1.0$, the optimal charting parameters $\left(n_{1}, n_{2}, L, L_{1}, L_{2}, L_{3}\right)=(2,6,1.3830,5.2804,2.0239,9)$ yield the lowest $\mathrm{EARL}_{1}=3.13$, when the process parameters are known. With these optimal charting parameters, EARL $\mathrm{E}_{1}=$ $\{3.56,3.36,3.26,3.18,3.15\}$ for $m=\{30,50,80,200,500\}$, respectively. It is noticeable that the $\mathrm{EARL}_{1}$ for the estimated process parameters case is deviated from the known process parameters. However, the $\mathrm{EARL}_{1}$ value approaches to the $\mathrm{EARL}_{1}$ value which corresponds to the $m=+\infty$ when the number of Phase-I samples increased. These findings show that more than 80 Phase-I samples are required to reduce the effects of process parameters estimation when estimating the process parameters from the in-control PhaseI samples.

To illustrate the implementation of the proposed optimal charting parameters, Table 2 presents the optimal charting parameters for the known process parameters SDS chart based on minimising out-of-control ARL $\left(\mathrm{ARL}_{1}\right)$ and the corresponding $\mathrm{ARL}_{1} \mathrm{~s}$ for the same combinations of $(m, n)$ in Table 1. Here, the in-control ARL, i.e., $\mathrm{ARL}_{0}=370.4$ is intended. Note that $\delta=\{0.2,0.5,0.9,1.2,1.5,1.9\}$ are considered here to accommodate the $\left(\delta_{\min }, \delta_{\max }\right)$ that are considered in Table 1; i.e., $\delta \in\{0.2,0.5,0.9\}$ and $\delta \in$ $\{1.2,1.5,1.9\}$ are included in $\left(\delta_{\min }, \delta_{\max }\right)=(0.2,1.0)$ and $\left(\delta_{\min }, \delta_{\max }\right)=(1.0,2.0)$, respectively. In Table 1 , when $n=3$, $\delta_{\text {min }}=0.2$, and $\delta_{\max }=1.0$, the EARL $1=3.13$ is obtained using the optimal charting parameters $\left(n_{1}, n_{2}, L, L_{1}, L_{2}, L_{3}\right)=$ $(2,6,1.3830,5.2804,2.0239,9)$. Here, by using the same optimal charting parameters for $\delta=0.5$ (i.e. $\delta \in\left(\delta_{\min }, \delta_{\max }\right)$ ), it yields $\mathrm{ARL}_{1}=\{17.76,14.32,12.89,11.72,11.32\}$ when $m=\{30,50,80,200,500\}$ using the ScicolsLab program. It is observed that the $\mathrm{ARL}_{1}$ value is almost the same to those in Table 2 when $n=3$ and $\delta=0.5$, although the optimal charting parameters based on minimising $\mathrm{ARL}_{1}$ are different, i.e., $\left(n_{1}, n_{2}, L, L_{1}, L_{2}, L_{3}\right)=(2,6,1.3830,5.2804,2.1867,18)$ (see Table 2). This indicates that the optimal charting parameters obtained based on minimising $\mathrm{EARL}_{1}$ can be employed as long as $\delta \in\left(\delta_{\min }, \delta_{\max }\right)$, i.e., when the practitioners do not have knowledge to determine the exact process shift size in advance.

\section{Conclusions}

In the production and manufacturing industries, it is a typical situation where quality practitioners are undecided about the process shift size to be implemented. The findings showed that the performance criterion EARL is capable of tackling the random shift size situation. Furthermore, the results also revealed that the performance of the SDS chart was adversely affected by process parameters estimation. This was proven when more than 80 Phase-I samples were needed for the chart 
TABLE 2: Optimal charting parameters $\left(n_{1}, n_{2}, L, L_{1}, L_{2}, L_{3}\right)$ and the corresponding $\mathrm{ARL}_{1} \mathrm{~s}$ for $n=\{3,4,5,6\}$ with different combinations of $(m, \delta)$ when $\mathrm{ARL}_{0}=370.4$.

\begin{tabular}{|c|c|c|c|c|c|c|c|c|c|c|c|c|c|}
\hline \multirow{2}{*}{$n$} & \multirow{2}{*}{$\delta$} & \multirow{2}{*}{$n_{1}$} & \multirow{2}{*}{$n_{2}$} & \multirow{2}{*}{$L$} & \multirow{2}{*}{$L_{1}$} & \multirow{2}{*}{$L_{2}$} & \multirow{2}{*}{$L_{3}$} & \multicolumn{6}{|c|}{$m$} \\
\hline & & & & & & & & 30 & 50 & 80 & 200 & 500 & $+\infty$ \\
\hline \multirow{6}{*}{3} & 0.2 & 2 & 6 & 1.3830 & 5.2804 & 2.4572 & 68 & 247.22 & 168.88 & 136.75 & 110.56 & 101.56 & 96.01 \\
\hline & 0.5 & 2 & 6 & 1.3830 & 5.2804 & 2.1867 & 18 & 16.68 & 13.35 & 12.03 & 10.99 & 10.63 & 10.41 \\
\hline & 0.9 & 2 & 6 & 1.3830 & 5.2804 & 1.9945 & 8 & 2.83 & 2.72 & 2.67 & 2.62 & 2.60 & 2.59 \\
\hline & 1.2 & 2 & 4 & 1.1503 & 5.0443 & 2.0178 & 4 & 1.64 & 1.62 & 1.60 & 1.59 & 1.58 & 1.58 \\
\hline & 1.5 & 2 & 3 & 0.9674 & 4.9920 & 2.0523 & 3 & 1.24 & 1.23 & 1.23 & 1.22 & 1.22 & 1.22 \\
\hline & 1.9 & 2 & 3 & 0.9674 & 4.9920 & 2.0523 & 3 & 1.06 & 1.06 & 1.06 & 1.05 & 1.05 & 1.05 \\
\hline \multirow{6}{*}{4} & 0.2 & 3 & 8 & 1.5341 & 5.1956 & 2.3954 & 60 & 149.45 & 113.15 & 95.73 & 80.45 & 75.01 & 71.62 \\
\hline & 0.5 & 3 & 8 & 1.5341 & 5.1956 & 2.0910 & 15 & 9.65 & 8.45 & 7.93 & 7.49 & 7.33 & 7.23 \\
\hline & 0.9 & 3 & 6 & 1.3830 & 5.2804 & 2.0153 & 6 & 2.12 & 2.07 & 2.04 & 2.02 & 2.01 & 2.00 \\
\hline & 1.2 & 3 & 4 & 1.1503 & 5.0443 & 2.0185 & 3 & 1.34 & 1.33 & 1.32 & 1.32 & 1.32 & 1.31 \\
\hline & 1.5 & 3 & 3 & 0.9674 & 4.9920 & 2.0086 & 2 & 1.10 & 1.10 & 1.10 & 1.09 & 1.09 & 1.09 \\
\hline & 1.9 & 3 & 3 & 0.9674 & 4.9920 & 2.0086 & 2 & 1.01 & 1.01 & 1.01 & 1.01 & 1.01 & 1.01 \\
\hline \multirow{6}{*}{5} & 0.2 & 4 & 10 & 1.6449 & 5.1247 & 2.3394 & 55 & 108.31 & 84.83 & 73.06 & 62.63 & 58.92 & 56.60 \\
\hline & 0.5 & 3 & 10 & 1.2816 & 5.1041 & 2.1216 & 12 & 6.64 & 6.05 & 5.78 & 5.54 & 5.46 & 5.40 \\
\hline & 0.9 & 3 & 8 & 1.1503 & 5.0443 & 2.0186 & 5 & 1.73 & 1.71 & 1.69 & 1.68 & 1.68 & 1.67 \\
\hline & 1.2 & 4 & 4 & 1.1503 & 5.0443 & 2.0615 & 3 & 1.20 & 1.19 & 1.19 & 1.18 & 1.18 & 1.18 \\
\hline & 1.5 & 4 & 4 & 1.1503 & 5.0443 & 1.9647 & 2 & 1.05 & 1.04 & 1.04 & 1.04 & 1.04 & 1.04 \\
\hline & 1.9 & 4 & 3 & 0.9674 & 4.9920 & 2.0363 & 2 & 1.00 & 1.00 & 1.00 & 1.00 & 1.00 & 1.00 \\
\hline \multirow{6}{*}{6} & 0.2 & 4 & 12 & 1.3830 & 5.2804 & 2.3744 & 44 & 84.09 & 66.77 & 58.05 & 50.35 & 47.62 & 45.91 \\
\hline & 0.5 & 4 & 12 & 1.3830 & 5.2804 & 2.0727 & 11 & 5.02 & 4.69 & 4.54 & 4.40 & 4.35 & 4.32 \\
\hline & 0.9 & 4 & 8 & 1.1503 & 5.0443 & 2.0178 & 4 & 1.50 & 1.48 & 1.47 & 1.46 & 1.46 & 1.46 \\
\hline & 1.2 & 5 & 5 & 1.2816 & 5.1041 & 2.0235 & 3 & 1.12 & 1.11 & 1.11 & 1.11 & 1.11 & 1.11 \\
\hline & 1.5 & 5 & 4 & 1.1503 & 5.0443 & 1.9954 & 2 & 1.02 & 1.02 & 1.02 & 1.02 & 1.02 & 1.02 \\
\hline & 1.9 & 5 & 3 & 0.9674 & 4.9920 & 2.0527 & 2 & 1.00 & 1.00 & 1.00 & 1.00 & 1.00 & 1.00 \\
\hline
\end{tabular}

with the estimated process parameters to behave similarly like the one with known process parameters. Therefore, future research works can consider the optimal charting parameters by minimising $\mathrm{EARL}_{1}$ for the SDS chart when the process parameters are estimated.

\section{Data Availability}

The ScicosLab programs to compute the average run length and expected average run length of the synthetic double sampling chart can be requested from the author.

\section{Conflicts of Interest}

The author declares that there are no conflicts of interest regarding the publication of this paper.

\section{Acknowledgments}

This research is supported by the Universiti Kebangsaan Malaysia, Geran Galakan Penyelidik Muda, GGPM-2017062.

\section{References}

[1] S. Shang, Q. Zhou, M. Liu, and Y. Shao, "Sample size calculation for controlling false discovery proportion," Journal of Probability and Statistics, vol. 2012, Article ID 817948, 13 pages, 2012.

[2] D. C. Montgomery, A Modern Introduction, John Wiley \& Sons, New York, NY, USA, 7th edition, 2013.

[3] M. B. C. Khoo, H. C. Lee, Z. Wu, C.-H. Chen, and P. Castagliola, "A synthetic double sampling control chart for the process mean," IIE Transactions, vol. 43, no. 1, pp. 23-38, 2010.

[4] N. A. Saleh and M. A. Mahmoud, "Accounting for phase I sampling variability in the performance of the MEWMA control chart with estimated parameters," Communications in Statistics-Simulation and Computation, vol. 46, no. 6, pp. 43334347, 2017.

[5] W. H. Woodall and D. C. Montgomery, "Some current directions in the theory and application of statistical process monitoring," Journal of Quality Technology, vol. 46, no. 1, pp. 78-94, 2014.

[6] S. Psarakis, A. K. Vyniou, and P. Castagliola, "Some recent developments on the effects of parameter estimation on control charts," Quality and Reliability Engineering International, vol. 30, no. 8, pp. 1113-1129, 2014.

[7] P. Castagliola, P. E. Maravelakis, and F. O. Figueiredo, "The EWMA median chart with estimated parameters," Institute of Industrial Engineers (IIE). IIE Transactions, vol. 48, no. 1, pp. 66-74, 2016. 
[8] S. Du, X. Yao, and D. Huang, "Engineering model-based Bayesian monitoring of ramp-up phase of multistage manufacturing process," International Journal of Production Research, vol. 53, no. 15, pp. 4594-4613, 2015.

[9] S. Chakraborti, "Run length distribution and percentiles: The shewhart Xc̄hart with unknown parameters," Quality Engineering, vol. 19, no. 2, pp. 119-127, 2007.

[10] H. W. You, M. B. C. Khoo, M. H. Lee, and P. Castagliola, "Synthetic double sampling $\mathrm{X}$ chart with estimated process parameters," Quality Technology and Quantitative Management, vol. 12, no. 4, pp. 579-604, 2016.

[11] Y. Ou, Z. Wu, K. M. Lee, and S. Chen, "An optimal design algorithm of the SPRT chart for minimizing weighted ATS," International Journal of Production Economics, vol. 139, no. 2, pp. 564-574, 2012.

[12] W. L. Teoh, J. K. Chong, M. B. C. Khoo, P. Castagliola, and W. C. Yeong, "Optimal Designs of the Variable Sample Size XfiChart Based on Median Run Length and Expected Median Run Length," Quality and Reliability Engineering International, vol. 33, no. 1, pp. 121-134, 2017. 


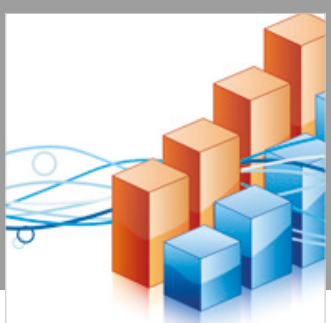

Advances in

Operations Research

\section{-n-m}
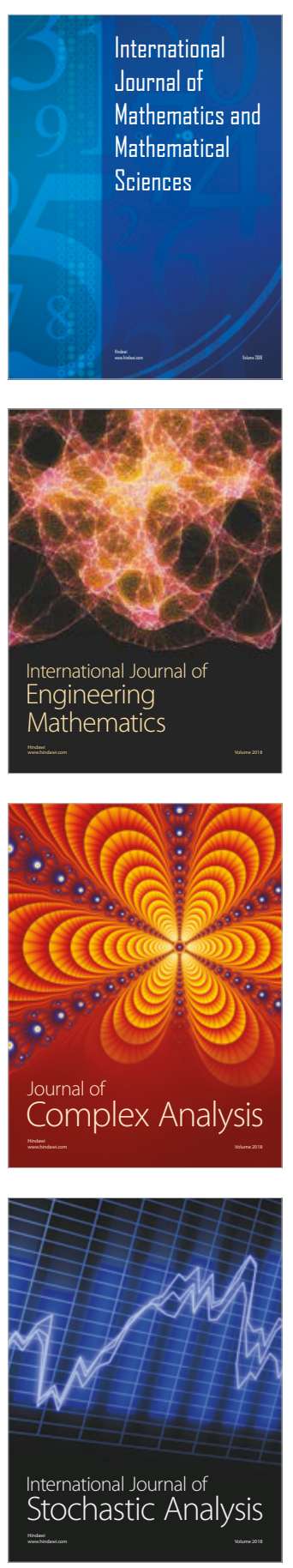
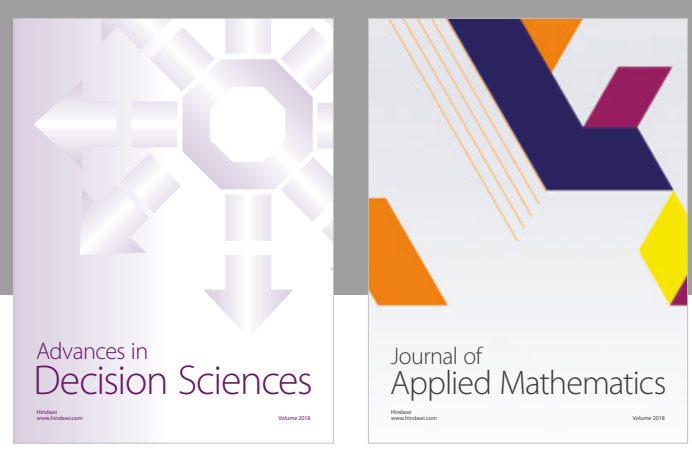

Journal of

Applied Mathematics
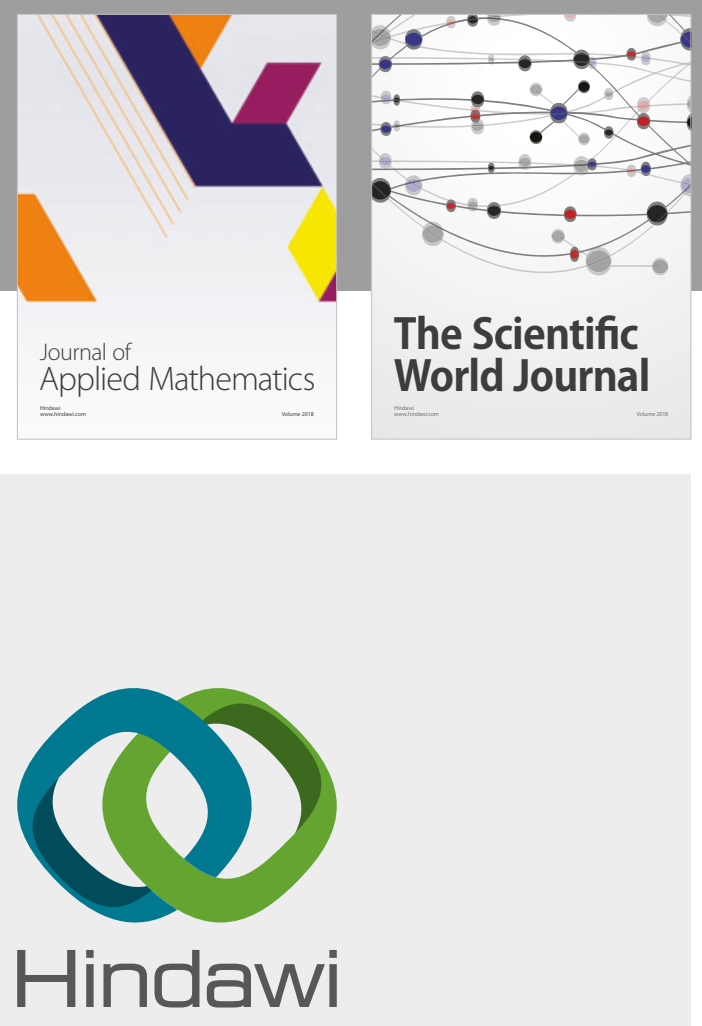

Submit your manuscripts at

www.hindawi.com

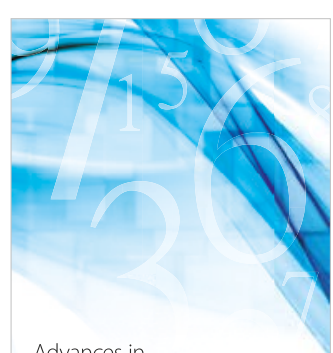

Advances in
Numerical Analysis
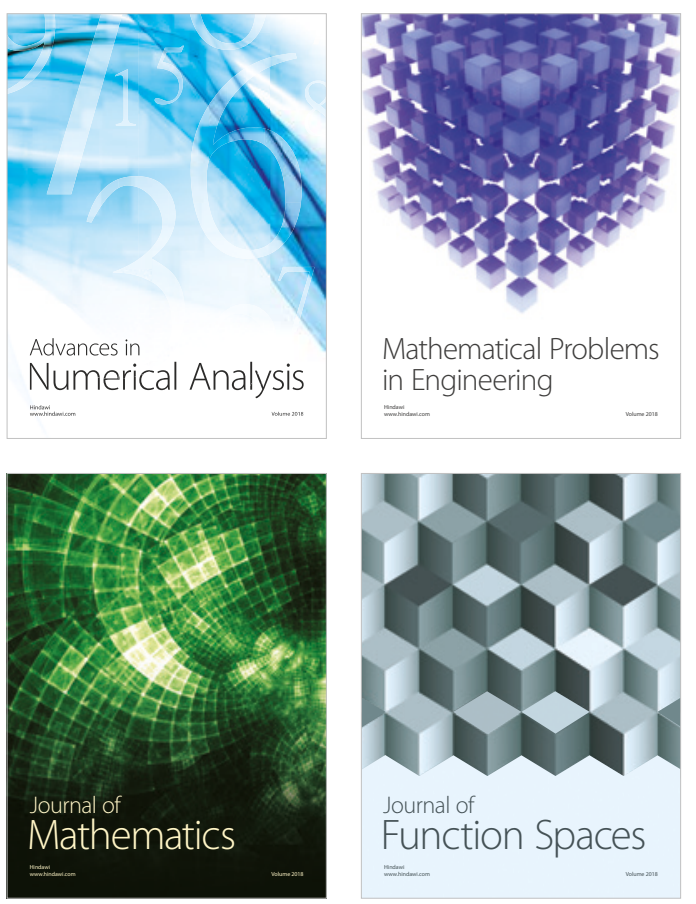

Mathematical Problems in Engineering

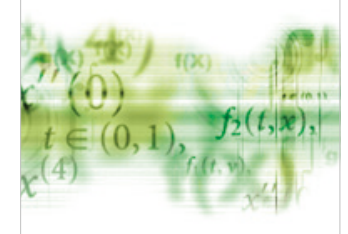

International Journal of

Differential Equations

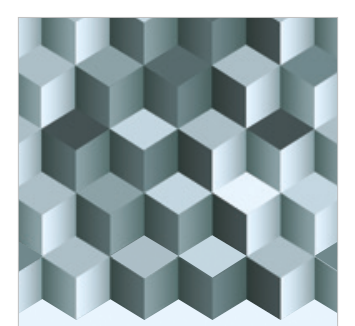

Journal of

Function Spaces

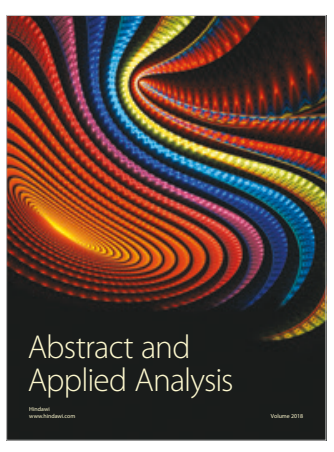

The Scientific

World Journal

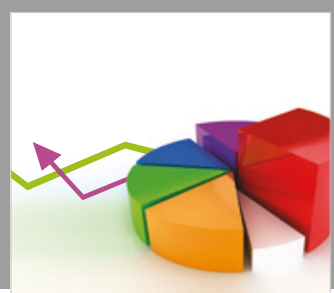

Journal of

Probability and Statistics
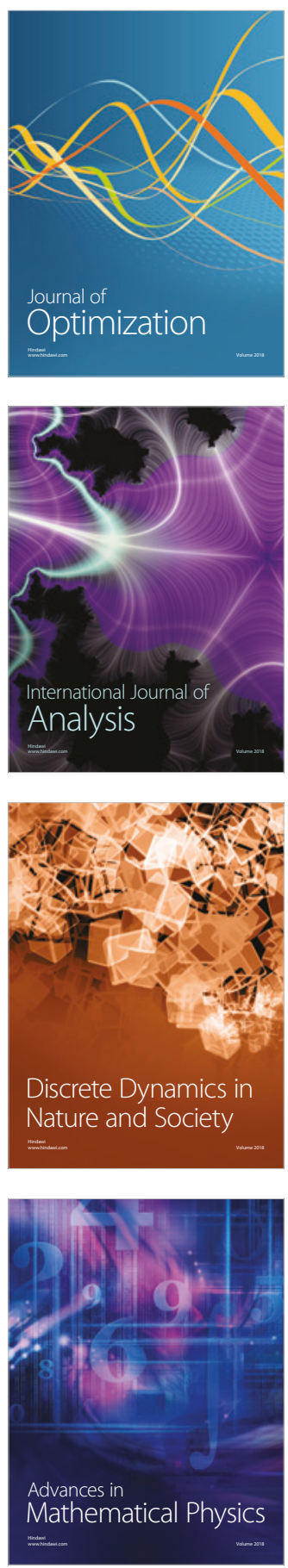